\title{
LEPROSY ELIMINATION: PROGRESS AND CHALLENGES IN NIGERIA; KADUNA STATE TB AND LEPROSY CONTROL PROGRAMME AS A CASE STUDY
}

\section{${ }^{1 *}$ Gidado Mustapha, ${ }^{1}$ Obasanya Joshua Olusegu, ${ }^{1}$ Sani Mustapha. ${ }^{1}$ Adesigbin Clement, ${ }^{2}$ Tahir Dahiru. ${ }^{3}$ Gagere J, ${ }^{4}$ Adejumo Adeleji Olusola}

\author{
${ }^{1}$ National TB \& Leprosy Training Centre, Zaria. \\ ${ }^{2}$ Netherlands Leprosy Relief, Terdaow House, Jos, Nigeria \\ ${ }^{3}$ Kaduna State TB \& Leprosy Control Programme \\ ${ }^{4}$ Lagos State University Teaching Hospital (LASUTH) Ikeja Lagos \\ :*E-mail: gidadomansu@yahoo.com.au
}

\begin{abstract}
The study aims at describing the achievements and challenges of Leprosy control in Kaduna State using appropriate indicators. The study was a five year (2004-2008) retrospective review of the Leprosy records and annual reports of all the twenty three LGAs in Kaduna State. Various Leprosy indicators were calculated and presented in different graphic presentations. Focus group discussions were organised with the aim of identifying current challenges of Leprosy control in the State. There was a decline in the new Leprosy cases detected annually from 226 cases in 2004 to 140 cases in 2008. The prevalence rate ranged between $0.3-0.4$ per 10,000 population within the five year period. The proportion of children among new cases dropped from 12\% in 2004 to 5\% in 2007 and increased to 9\% in 2008. Grade 2 disability among new cases was very high (between 21\%-27\%) within the same period. Leprosy elimination target has been achieved in Kaduna State, but new cases with high proportion of children and WHO grade 2 disability were still been reported
\end{abstract}

Key words: Leprosy; Elimination; Leprosy indicators

\section{Introduction}

Despite availability of care, Leprosy is still associated with severe disability and strong social stigma. The resolution to 'eliminate Leprosy as a public health problem' by the year 2000 was passed at the $44^{\text {th }}$ World Health Assembly in 1991. Elimination of Leprosy is defined as reducing the prevalence of Leprosy to less than one case per 10,000 population (WHO, 1991). The World Health Organization (WHO) developed elimination strategies based on increasing geographical coverage of Multi Drug Therapy (MDT) and patient's accessibility to treatment. Intensive "Leprosy Elimination Campaigns" were held in many countries. The Global target for Leprosy was however reached in 2000 with the world prevalence of less than 600,000 cases. (Jan and Habbema, 2007)

Nigeria adapted the global elimination target with strategies based on early case finding, provision of MDT to all detected cases, ensuring case holding and integration of Leprosy services into general health care services. (FMOH, 2008a). The national elimination target was achieved by end of 1998 and at the end of 2007, all the States in Nigeria except Taraba State has attained the Leprosy elimination target. (FMOH, 2008b)

The Leprosy control program in Kaduna State is combined with tuberculosis control program and is integrated into general health care services. Leprosy service is provided in 80 primary health care facilities located in all the 23 LGAs of the State. The target set for Leprosy elimination in Kaduna State was achieved in 2001 and currently, the prevalence rate of Leprosy is 0.3/10,000 population in the State. (Kaduna State MOH, 2007)

\section{Materials and Methods}

The study was retrospective with review of five years (2004 to 2008) program data. The Local Government Leprosy Central registers of all the 23 LGAs were reviewed and compared with the quarterly and annual statistical report of the LGAs. The LG Leprosy register summarises data of all patients registered in the health facilities located within the LGA. Every patient's information is summarized in one row. While the quarterly statistical forms summarises the registered cases quarterly and provided opportunity for the calculation of the different indicators. Also reviewed were the annual reports of the State TB \& Leprosy Control Program between 2004 and 2008.

The indicators used were mainly those reported by the program on quarterly and annual bases using the quarterly/annual statistical form (NTBLCP/LEP3). They are: prevalence rate, case detection rate, proportion of multi bacillary patients among new cases, proportion of Grade 2 disability among new cases and proportion of children among new cases. The prevalence and case detection rates are expressed per 10,000 populations, while all other proportions are in percentages. 
Two focus group discussions were conducted among the LG TB \& Leprosy supervisors during one of their quarterly meetings in order to identify the challenges encountered after achieving the Leprosy elimination target in the State. Statistical analysis was done using excel and results are presented in graghs. The transcripts of the FGDs were read several times in order to identify themes emerging from the discussions. Verbatim quotes were used to capture the perspective of the discussants and also consensus and disagreements based on the groups involved were identified.

\section{Results}

The number of Leprosy cases detected within the period under review in the state was 956; the cases were not evenly distributed in the state. Majority of the cases (32\%) were reported in Zaria LGA while 18 LGAs contributed $41 \%$ of the total new cases seen. Figure 1.

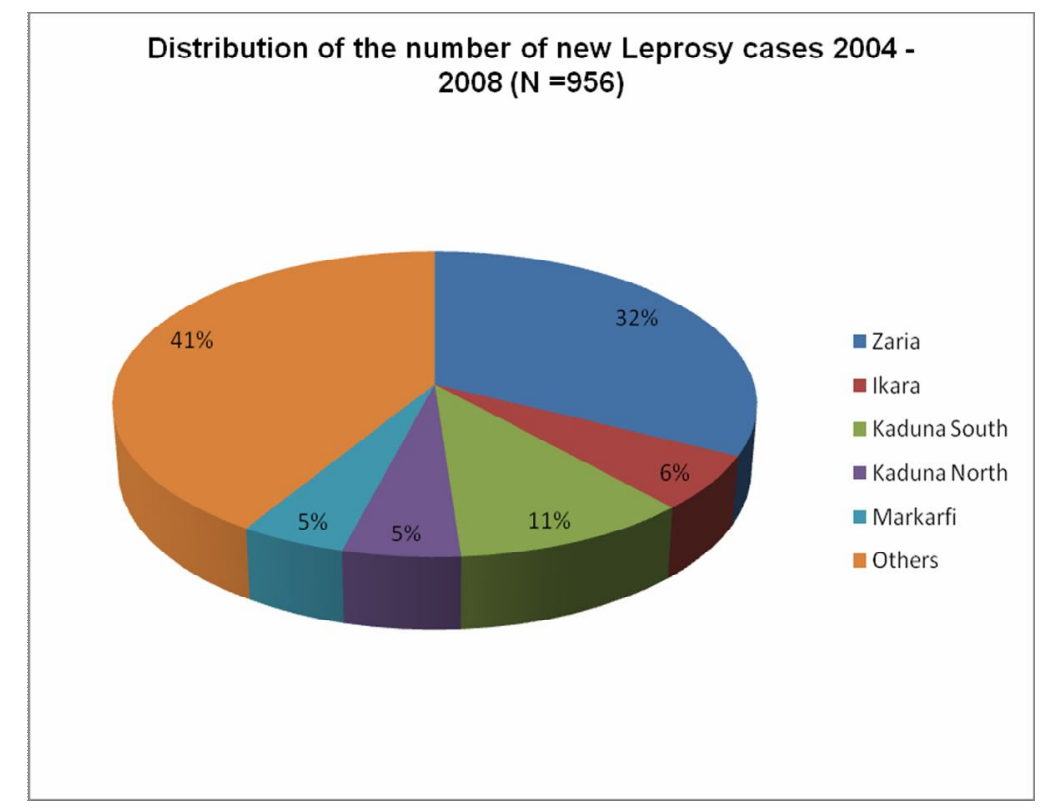

Figure 1: Distribution of the number of new Leprosy cases 2004-2008

There was a decline in the number of new cases detected from 226 cases in 2004 to 140 cases in 2008. (Figure 2). Both the prevalence and case detection rates also declined from $0.4 \%$ in 2004 to $0.3 \%$ at the end of 2008. (Figure 3). All LGAs located in the State achieved the Leprosy elimination target with the exception of Zaria LGA which had a prevalence rate of $1.4 / 10,000$ population

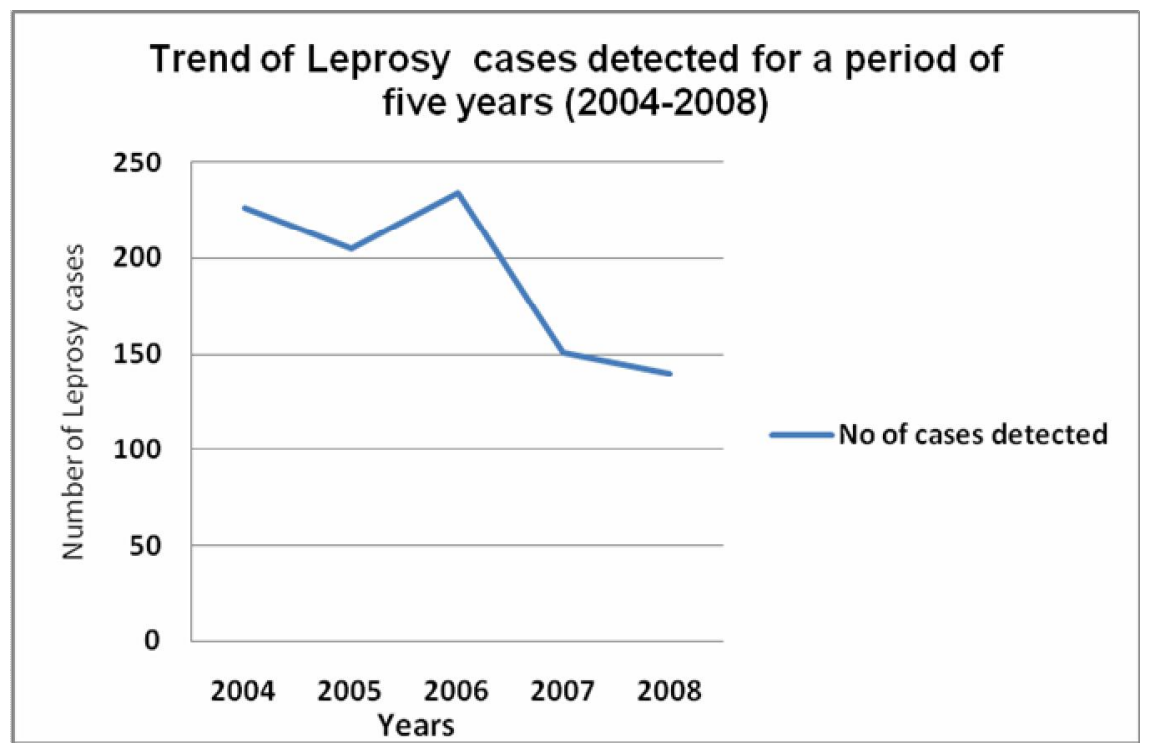

Figure 2: Trend of Leprosy cases detected for a period of five years 
This review also showed a decline in the proportion of children among new cases from $12 \%$ in 2004 to $5 \%$ in 2007, however there was an increase in 2009 to $9 \%$. The W.H.O Grade 2 Disability among new cases was high and ranged between $21 \%$ and $27 \%$ within the five year period. Most of the patients were multi bacillary (MB) Leprosy case and the proportion of the MB has been on the increase from $83 \%$ in 2004 to $92 \%$ in 2008 (Figure 4).

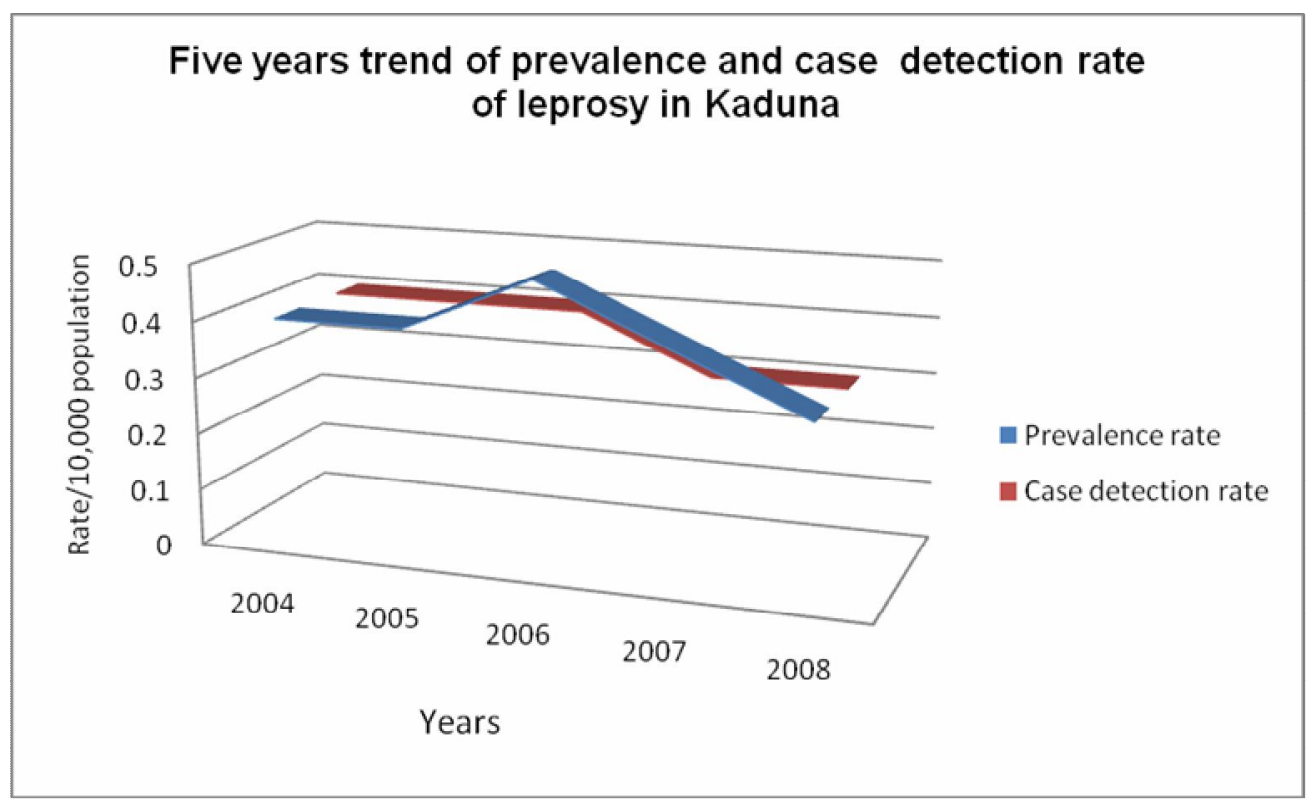

Figure 3: Five years trend of prevalence and case detection rate of Leprosy in Kaduna

Five year trend of Leprosy indicators in Kaduna State (2004-2008)

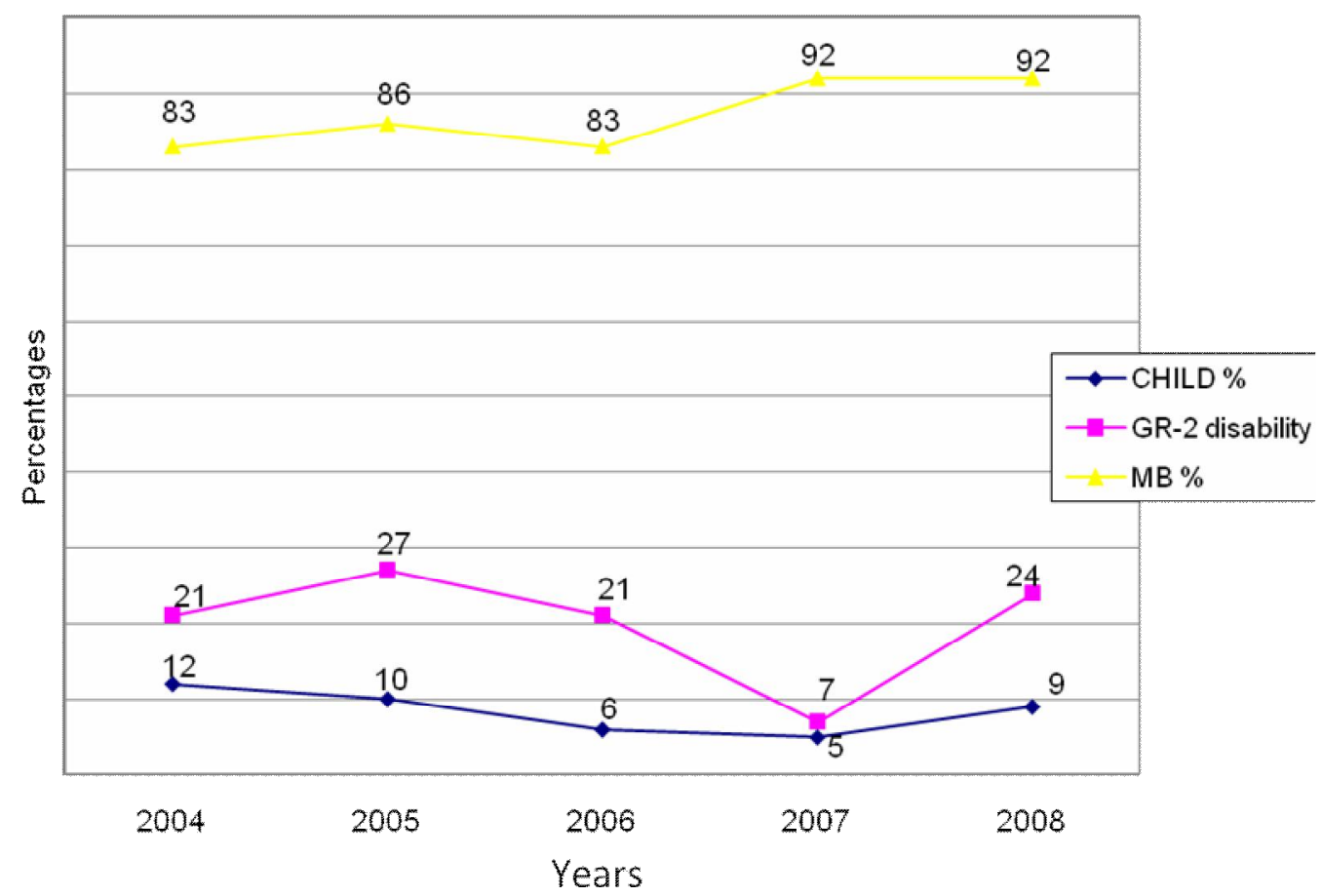

Figure 4: Five year trend of Leprosy indicators in Kaduna State 
During the focus group discussions held, the LG TB \& Leprosy supervisors and the State team identified the following as challenges facing Leprosy control in Kaduna State after reaching the elimination target.

- $\quad$ Lack of adequate skills in detecting and management of Leprosy patients among general health workers

- $\quad$ Poor recording, especially skin charting on the Leprosy cards

- $\quad$ Poor referral linkages of Leprosy patients

- $\quad$ Non involvement of secondary and tertiary health facilities.

- $\quad$ Lack of comprehensive Leprosy rehabilitation activities

- $\quad$ Impact of TB and HIV program activities on Leprosy as most health workers are busy with TB and HIV program.

- Low government commitment to Leprosy control in the state

\section{Discussion}

The review showed a declining trend of both prevalence and case detection rate of Leprosy (including absolute new cases in the last five years) in Kaduna State. The prevalence rate in the State has consistently remained below one case per 10,000 population, with the exception of Zaria LGA with a prevalence of $1.4 / 10,000$. This finding is comparable to the national prevalence of $0.4 / 10,000$ population but defer on the ground that at the national level, the new cases detected yearly has remained stable between 4,500-6,000 (FMOH, 2008b). However similar picture is reported globally with a decline in new cases detected from 668,658 in 2001 to 174,118 cases in 2006. (Salvatore and Enrico, 2008).

The high prevalence rate of 1.4/10,000 population reported in Zaria LGA could be attributed to the pooling effect of the National TB \& Leprosy Training Centre located in Zaria which serves as a referral centre to many states in Northern Nigeria. This suggests that many of the reported cases are not from Zaria LGA.

Leprosy elimination target using prevalence rate has been a subject of debate for years, because of its sensitivity to treatment duration and the fact that annual cases detected has not declined globally since 1985. (Abraham et al, 2004). The prevalence of Leprosy declined very fast with the introduction of MDT because of the reduction in the duration of treatment of Leprosy from over five years to just 12 months in the case of MB. As at the end of 2006 only six countries in the world are yet to reach the elimination goal (Brazil, Democratic Republic of the Congo, Madagascar, Mozambique, Nepal and United Republic of Tanzania).(Fine, 2006)

The second challenge is the lack of common understanding of the definition of "elimination" between technical groups and policy makers. The WHO defined Leprosy elimination in terms of reduction in the prevalence of Leprosy to a level that it ceases to be a 'public health problem' is different from the concept of disease elimination 'defined as a reduction to zero level in the incidence of infection caused by a specific agent in a defined geographical area as a result of deliberate efforts; but requires measure to prevent reestablishment of transmission (Jan and Habbema 2007). Eradication on the other hand suggests zero incidence and zero transmission of disease agents. Leprosy eradication was based on an assumption that after achieving elimination target, Leprosy would die out over a period of time, provided all Leprosy services continue to be available. (Noordeen, 2007a). This assumption has however contributed to low government commitment experienced in many countries after achieving the elimination target.

The Grade 2 disability (G2D) is an observable disability among new cases and is a measure of case finding, high grade 2 disability indicates late case detection in a community. This has been consistently high above 5\% in Kaduna state in the last five years (mean of 19.2\%). This figure is above both global and national figures of $2.2 \%$ and $14 \%$ respectively. (FMOH, 2008b; Anonymous, 2007)

While the child proportion (CP) among new cases indicates continuous transmission of Leprosy in the community. The mean child proportion in Kaduna was $8.4 \%$, which is slightly below the national figure of $10.8 \%$. (FMOH, 2008b).

Both indicators (G2D and CP) are very sensitive to total new cases detected (as the denominator) and they are used as measures of program performance. They must be interpreted with caution because they are less sensitive when case detection is low. (ILEP, 2001).

As a result of the issues surrounding the usage of these indicators, WHO proposed the use of "the rate of new cases with grade-2 disability per 100,000 population" as a global indicator. The new indicator is less influenced by operational factors; focuses attention on impairments and also stimulates improvement in case detection. (WHO, 2009). The goal of the 2011-2015 strategy is to reduce the rate of new cases with grade- 2 disability per 100,000 population by at least $35 \%$ by end of 2015 with a base line in 2010.(WHO, 2009).

One of the challenges identified during the focus group discussions was lack of sufficient knowledge and skills of health care workers. This may be due to the very low prevalence of Leprosy in the State. Some health workers may not encounter a Leprosy case for months, which may lead to loss of the required skill to establish the diagnosis. Noordeen stated that maintenance of skills among peripheral health workers, dealing with complications of Leprosy, disability and rehabilitation are the challenges that may be encountered after the achievement of Leprosy elimination target. (Noordeen, 2007b). Sustainability of Leprosy services depends largely on two factors: capacity building at peripheral levels and establishment of effective referral system. One recommendation has been the involvement of skin/dermatologist in Leprosy control with an effective referral system. (Ruchika, 2007; Srinivas, 2007). The implication for the Leprosy programme in Nigeria is to integrate Leprosy control activities into the Secondary and Tertiary health facilities for diagnosis and management of complicated Leprosy cases and strengthening of the referral system. Health workers at primary health care levels and private sector should be trained on how to suspect Leprosy based on clinical signs and refer to designated centres for confirmation, MDT administration and identification of early signs of Leprosy reaction. While health care workers at secondary and tertiary health facilities should be trained on diagnosis and management of complications of Leprosy. 


\section{References}

1. Abraham Meima, Jan H. Richadus \& J. Dik F. Habbema. (2004). Trends in Leprosy case detection worldwide since 1985. Leprosy Review. 75:19-33.

2. Anonymous. (2007). Leprosy Global Situation. Weekly Epidemiology Record, 82: 225-232.

3. Fine P. E. M.(2006). Global Leprosy statistics: a cause for pride, or frustration? Leprosy Review. 77:295-297

4. Federal Ministry of Health Abuja, (2008a) Department of Public Health. National TB \& Leprosy Control Workers Manual $5^{\text {th }}$ Edition,

5. Federal Ministry of Health Abuja, (2008b). Department of Public Health. National TB \& Leprosy Control programm, Annual report.

6. International Federation of Anti-Leprosy association (2001); interpretation of epidemiological indicators in Leprosy. ILEP Technical bulletin. London.

7. Jan Hendrik Richadus \& J. Dik F. Habbema. (2007). The impact of Leprosy control on the transmission of M. Leprae: is elimination being attained? Leprosy Rev. 78: 330-337

8. Kaduna State Ministry of Health, Nigeria.(2007) Kaduna State TB \& Leprosy Control Programm, Annual report.

9. Noordeen S.K. (2007a). Elimination of Leprosy: Success brings new challenges. Health Administrator, Vol. XVIII, 2: $15-17$

10. Noordeen S. K. (2007b). Role of dermatologist in Leprosy. Leprosy Review 78, 52-53

11. Ruchika Chandna.(2007) Role of dermatologist in Leprosy. Leprosy Rev 78, 43-44

12. Salvatore Noto \& Enrico Nunzi. (2008) Global and regional annual 'new case detection' of Leprosy reported by World Health Organisation. Leprosy review, 79, 124-127

13. Srinivas Prasanna Kumar Athreya. (2007). Role of dermatologist in Leprosy. Leprosy Rev 78, 40

14. World Health Assembly. (1991). Elimination of Leprosy; resolution of the $44^{\text {th }}$ World Health Assembly, World Health Organisation, Geneva, (resolution No WHA 44.9)

15. World Health Organisation. (2009). Enhanced Global Strategy for Further Reducing the Disease Burden due to Leprosy, 2011-2015. World Health Organisation, Geneva. 